\title{
Paenibacillus Isolated from Superficial Infection of the Left Knee Region in Middle-Aged Man
}

\author{
Mohamed Shaalan* (D), Ali Omer, Mohamed Elrih and Satish Kutty
}

Sligo University Hospital, Ireland

*Corresponding author: Mohamed Shaalan, Sligo University Hospital, Ireland

\begin{abstract}
Summary
49-year-old gentleman employed in a swimming pool as a lifesaver, has right anterior knee pain and swelling, aspiration was done by his general practitioner (GP) 4 days earlier and culture results showed streptococcus and he has been treated with an oral antibiotic with minimal response.

On admission, there is subcutaneous diffuse, fluctuant swelling anterior to the left patella measuring $3 \times 4 \mathrm{~cm}$, which is erythematous, warm and tender, the range of movements painful beyond 90 degrees. Temperature is 36.8 degrees, pulse rate 97 per minute. Haematological investigations revealed; CRP is 322 , WBC 20.15 , Neutrophils 18.88 , an incision and drainage was done in theatre under general anaesthesia, it was yellowish fluid $15 \mathrm{ml}$ which sent for culture and sensitivity and wound packed with a wick, IV flucloxacillin $2 \mathrm{gm}$ QDS started, after two days second look and washout, and wound closed. Culture results after 48 hours, Paenibacillus organism grew anaerobically and microbiologist advised to start triple antibiotic, Clindamycin, benzylpenicillin and Flucloxacillin, and patient fully recovered with normal blood results.
\end{abstract}

\section{Background}

Paenibacillus comprises bacterial species relevant to humans, animals, plants, and the environment, Many species of Paenibacillus produce antimicrobial compounds that are useful in medicine, Some species are pathogens to honeybees or other invertebrates; while others are occasional opportunistic infectors of humans. Several Paenibacillus species have been isolated from humans globally. Although the majority of these colonisations are not harmful to their host, some have demonstrated pathogenicity to humans. In almost every case, Paenibacillus infections are opportunistic and tend to infect immunocompromised people. Diseases or syndromes associated with Paenibacillus infection include chronic kidney disease [1], sickle cell disease [2], premature birth [3], Whipple's disease [4], hydrocephalus [5], skin cancer, chronic interstitial nephropathy, and acute lymphoblastic leukemia [6].

\section{Case Presentation}

49-year-old gentleman employed in swimming pool as lifesaver, presented to the emergency department with right anterior knee pain and swelling, aspiration was done by his general practitioner (GP) 4 days earlier and sample sent for culture and sensitivity (c/s) and culture results showed streptococcus and he has been treated with an oral antibiotic, (Flucloxacillin $500 \mathrm{mg}$ QDS) with minimal response.

On admission to orthopaedic via ED, there is subcutaneous diffuse, fluctuant swelling anterior to the left patella measuring $3 \times 4 \mathrm{~cm}$, which is erythematous, warm to touch and tender, the range of movements are full but painful beyond 90 degrees. Temperature is 36.8 degrees, pulse rate 97 per minute, and respiratory rate per minute, haematological investigations revealed; CRP is 322 , WBC 20.15, Neutrophils 18.88 , an incision and drainage was done in theatre under general anaesthesia, it was yellowish fluid $15 \mathrm{ml}$ which sent for culture and sensitivity, and wound packed with a wick, IV flucloxacillin $2 \mathrm{gm}$ QDS started and patient brought to the operation room after two days for second look and washout, and wound closed. During culture incubation, culture results after 48 hours, Paenibacillus organism grew anaerobically and as advised by microbiology department triple antibiotics was begun, Clindamycin 600 mg IV 12 days then oral $600 \mathrm{mg} 6$ weeks, benzylpeni-

Citation: Shaalan M, Omer A, Elrih M, Kutty S (2020) Paenibacillus Isolated from Superficial Infection of the Left Knee Region in Middle-Aged Man. Int Arch Orthop Surg 3:023. doi.org/10.23937/26434016/1710023

Accepted: December 19, 2020; Published: December 21, 2020

Copyright: (C) 2020 Shaalan M et al. This is an open-access article distributed under the terms of the Creative Commons Attribution License, which permits unrestricted use, distribution, and reproduction in any medium, provided the original author and source are credited 
Table 1: Antibiotic regimen.

\begin{tabular}{|l|l|l|l|l|}
\hline Name of antibiotic & Dose & Route & frequency & Duration \\
\hline Clindamycin & $600 \mathrm{mg}$ & IV & OD & 12 Days \\
\hline benzylpenicillin & $2.4 \mathrm{gm}$ & IV & QDS & 12 Days \\
\hline Flucloxacillin & $2 \mathrm{gm}$ & IV & QDS & 22 Days \\
\hline Clindamycin & $600 \mathrm{mg}$ & PO & OD & 45 Days \\
\hline Flucloxacillin & $1 \mathrm{gm}$ & PO & QDS & 35 Days \\
\hline
\end{tabular}

Table 2: Haematological results.

\begin{tabular}{|l|l|l|l|l|l|l|}
\hline Date & CRP & ESR & Hb & WBCS & Neutrophils & C/S \\
\hline $18 / 4 / 2018$ & 322 & & 13 & 20.15 & 18.88 & \\
\hline $22 / 4 / 2018$ & & & & & & Paenibacillus provencensis \\
\hline $26 / 4 / 2018$ & 37 & 57 & 11.3 & 6.99 & 4.29 & \\
\hline $5 / 5 / 2018$ & 6 & 26 & 13 & 4.86 & 2.64 & No Growth \\
\hline $7 / 5 / 2018$ & 2 & & 12.8 & 4.85 & 2.18 & \\
\hline
\end{tabular}

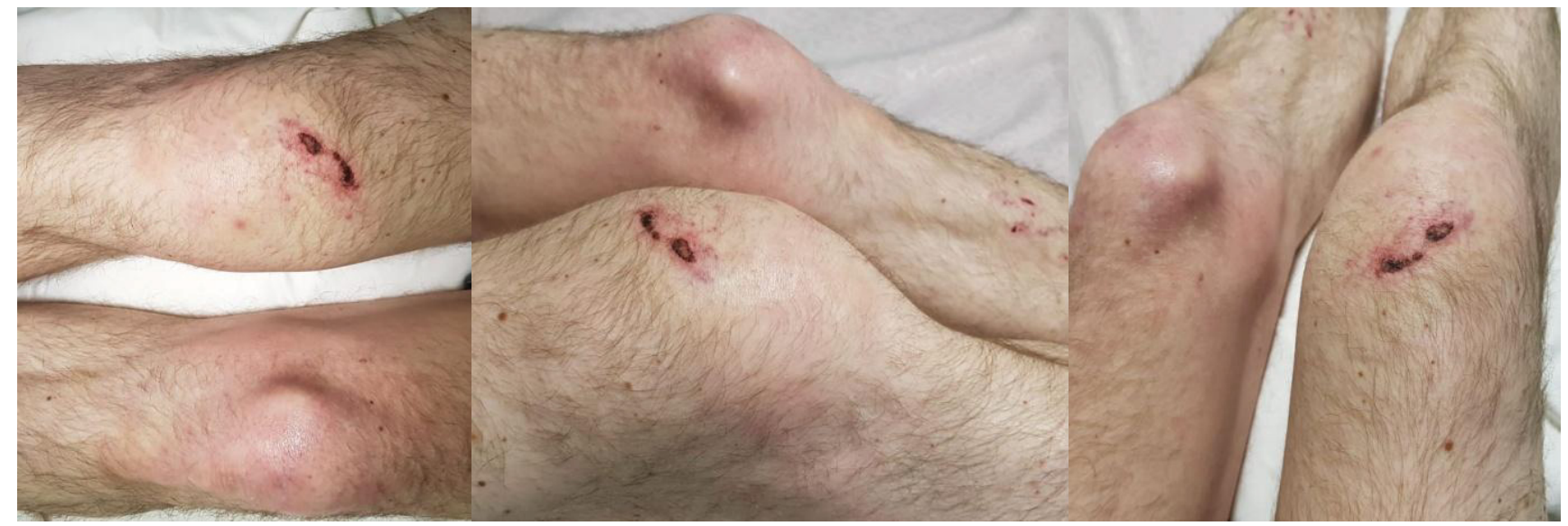

Figure 1: Knee pictures on admission.

cillin 2.4 gm 6 hourly 12 days and Flucloxacillin 2 gm 6 hourly 22 days then started oral 1 gm 6 hourly 5 weeks (Table 1).

A slow but definite response was noted.

US examination of the right knee done on the day following his admission showed no evidence of knee joint effusion, however a subcutaneous collection in keeping with cellulitis (Figure 1 and Figure 2).

\section{Results of Haematological Investigations}

Haematological results Table 2.

\section{Differential Diagnosis}

Skin and soft tissue infections are usually caused by Staphylococcus aureus (S. aureus) and Streptococcus pyogenes.

Thrombophlebitis, superficial Tender, palpable cord along affected vein often present. The presence or recent presence of intravenous catheter or needle also suggests this diagnosis.

Necrotizing fasciitis Initial findings are nonspecific and can be similar to those of cellulitis, Marked pain, of- ten out of proportion to the exam, and necrotic bullous change are clinical clues.

Deep vein thrombosis Tenderness of involved vein, history of prior deep venous thrombosis, prolonged immobility, or hypercoagulable state.

Lyme disease Residence in or travel to the endemic area, history of tick exposure, the involvement of sites that are unusual for bacterial cellulitis (e.g., axilla, popliteal fossa, or abdomen) are suggestive.

Dermatitis, (contact) Well-demarcated skin involvement, pruritus, and exposure history are suggestive.

Insect bites and stings History of insect exposure and pruritus. Often diagnosed on clinical grounds alone.

Fixed drug reactions History of similar reaction with prior exposure to the same drug; well-demarcated area of involvement; itching, burning; involvement of lips and/or genitalia.

Eosinophilic cellulitis (Wells syndrome), a short prodrome of itching and burning may precede onset of single or multiple lesions. Recurrence is common and resolution of each episode may occur over weeks. 


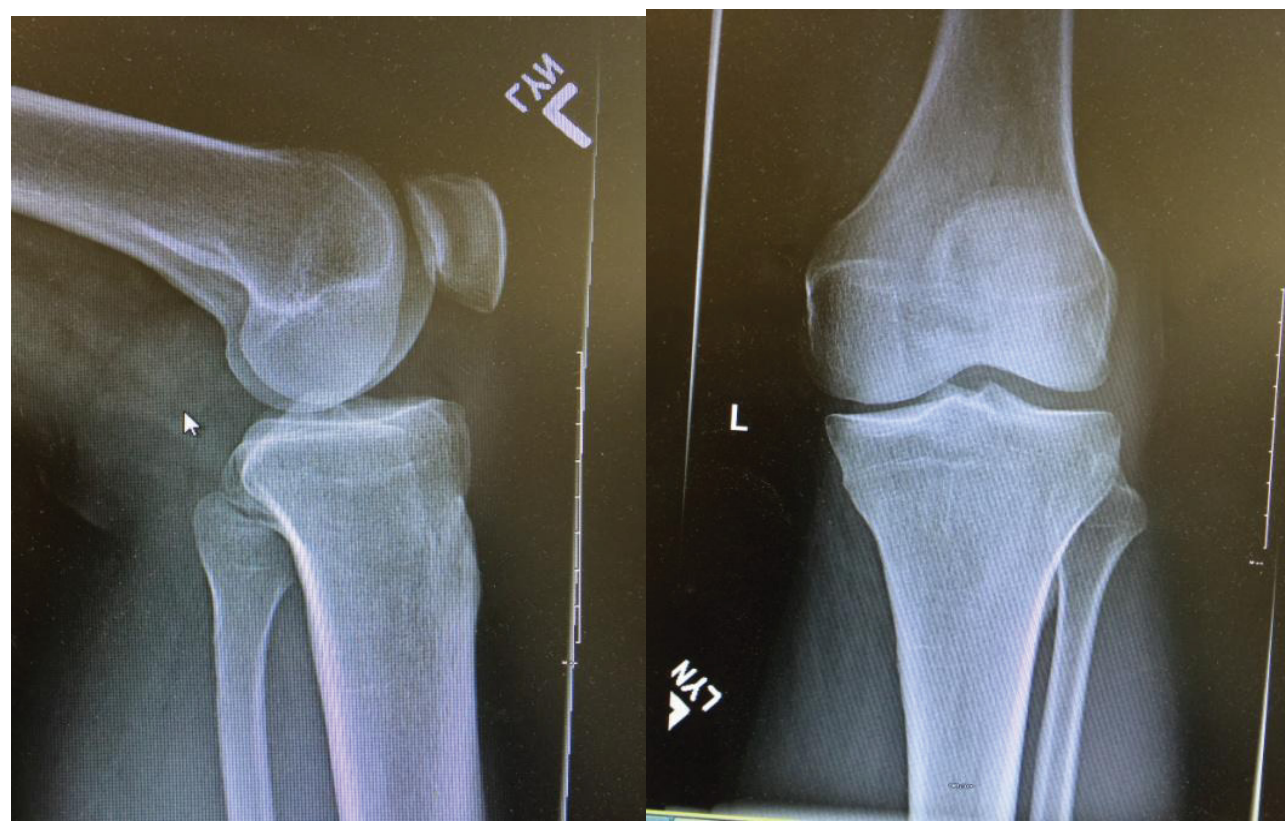

Figure 2: X-Ray done on admission and no abnormality seen.

Sweet syndrome, Well-defined erythematous plaques with a mammillated surface are typical.

Familial Mediterranean fever, a positive family history, recurrent episodes, association with syndrome of fever, serositis, and lower extremity involvement with erysipelas like lesions are suggestive.

Lymphedema, History of malignancy, previous surgery, radiation therapy, travel to endemic filariasis area, or family history of lymphedema. Painless unilateral swelling of extremity; non-pitting oedema; skin changes. Positive Stemmer sign (inability to tent the skin on the dorsum of the digits). Symptoms of limb heaviness or weakness.

\section{Results}

The cellulitis completely resolved with no recurrence of inflammation till now, the patient has regained movement in the right knee, he developed normal gait and has return work, haematological investigations done and returned normal. One should express caution and maintain vigilance while dealing with infection caused by paenibacillus.

\section{Discussion}

Paenibacillus are occasional opportunistic infectors of humans. Several Paenibacillus species have been isolated from humans globally. Although the majority of these colonisations are not harmful to their host, some have demonstrated pathogenicity to humans. In our case infection was slowly responding to the antibiotic therapy, we should put in mind these rare infections especially in patients working in swimming pools, we are not sure about the correlation between the media of swimming pools and paenibacillus infection.

\section{Conclusion}

Paenibacillus only rarely appears to be pathogenic to humans. Most of its species non-pathogenic. Paenibacillus is an occasional opportunistic infector of humans. Several Paenibacillus species have been isolated from humans globally. Although the majority of these colonisations are not harmful to their host, some have demonstrated pathogenicity to humans such as in our case.

\section{References}

1. Padhi S, Dash M, Sahu R, Panda $P$ (2013) Urinary tract infection due to Paenibacillus alvei in a chronic kidney disease: A rare case report. J Lab Phys 5: 133-135.

2. Reboli AC, Bryan CS, Farrar WE (1989) Bacteremia and infection of a hip prosthesis caused by Bacillus alvei. J Clin Microbiol 27: 1395-1396.

3. DeLeon SD, Welliver RC Sr (2016) Paenibacillus alvei sepsis in a neonate. Pediatr Infect Dis J 35: 358.

4. Roux V, Fenner L, Raoult D (2008) Paenibacillus provencensis sp. nov., isolated from human cerebrospinal fluid, and Paenibacillus urinalis sp. nov., isolated from human urine. Int J Syst Evol Microbiol 58: 682-687.

5. Bosshard PP, Zbinden R, Altwegg M (2002) Paenibacillus turicensis sp. nov., a novel bacterium harbouring heterogeneities between 16S rRNA genes. Int J Syst Evol Microbiol 52: 2241-2249.

6. Roux V, Raoult D (2004) Paenibacillus massiliensis sp. nov., Paenibacillus sanguinis sp. nov. and Paenibacillus timonensis sp. nov., isolated from blood cultures. Int J Syst Evol Microbiol 54: 1049-1054. 